\title{
Multiple Objective Programming with \\ Piecewise Linear Functions
}

\author{
Stefan Nickel \\ and \\ Malgorzata M. Wiecek * \\ Fachbereich Mathematik \\ Universität Kaiserslautern \\ 67653 Kaiserslautern \\ Germany
}

September 23, 1996

\begin{abstract}
An approach to generating all efficient solutions of multiple objective programs with piecewise linear objective functions and linear constraints is presented. The approach is based on the decomposition of the feasible set into subsets, referred to as cells, so that the original problem reduces to a series of linear multiple objective programs over the cells. The concepts of cell-efficiency and complex-efficiency are introduced and their relationship with efficiency is examined. A generic algorithm for finding efficient solutions is proposed. Applications in location theory as well as in worst case analysis are highlighted.
\end{abstract}

\footnotetext{
${ }^{*}$ On sabbatical leave from the Department of Mathematical Sciences, Clemson University, Clemson, SC 29634-1907, U.S.A., e-mail: wmalgor@clemson.edu
} 


\section{Introduction}

Theory and methodology of multiple objective linear programming (MOLP) have been already intensively studied and aplied to a variety of decision making problems. In particular, the efficient set of those problems can be now easily computed by means of various algorithms. See [Yu and Zeleny, 1975], [Gal, 1977], [Isermann, 1977], [Ecker et al., 1980], [Steuer, 1986], [Armand, 1993], [Wiecek and Zhang, 1996] for different methodologies of finding the efficient set. Similarly, problems with convex objective functions over a convex feasible set have been treated, although their efficient set is in general not available in such an elegant form as it is for linear problems (see [Haimes and Chankong, 1983]). Motivated by applications, in this paper we examine possibly a simplest class of convex problems, i.e. multiple objective problems with piecewise linear objective functions and linear constraints, for which we develop an MOLP-type description of the efficient set and propose an approach to finding this set.

Single or multiple objective piecewise linear programs have been also studied by other authors. Fourer ([Fourer, 1985], [Fourer, 1988], [Fourer, 1992]) extended the simplex method for linear programming to permit the minimization of a convex piecewise linear function. Achary ([Achary, 1989]) developed a simplex type method for a transportation problem with two objective functions: a piecewise linear convex function and a piecewise constant function representing cost and time respectively.

Multiple objective piecewise linear programs (MOPLPs) specifically arise in location problems, where given a number of exisiting facilities a new facility has to be located so that some objectives are optimized. The objectives are often in the form of piecewise linear functions due to the fact that typically the sum (or the maximum) of weighted distances from the existing facilites to the new facility is chosen as a criterion. Moreover, these distances are often derived from norms with a polyhedral unit ball. See [Durier and Michelot, 1986], [Pelegrin and Fernandez, 1988], [Hamacher and Nickel, 1993], and [Puerto and Fernandez, 1994] for various MOPLP models in location theory.

Another area of application is multiple objective worst case analysis. If, for example, the coefficients of a linear objective function should represent cost of introducing a new product on the market, their exact numerical values are usually unknown but may be available from market analysis in the form of a collection of vectors. Under this uncertainty, the worst case analysis dictates that the resulting objective function be determined through the maximization over the linear functions corresponding to the vectors in the collection and hence the objective function becomes a piecewise linear function.

Furthermore, in order to obtain a more structured description of the efficient set of convex multiple objective programs one may consider approximating the convex objective functions by piecewise linear functions. Such an approximation method including error bound analysis was proposed by [Burkard et al., 1991].

We note that as a single objective piecewise linear program can be transformed into a linear program (see [Murty, 1983], p. 18), then also an MOPLP can be converted into a multiple objective linear program for which solution techniques are available, as mentioned before. Such a transformation, however, would significantly increase the number of variables and 
constraints. The approach presented in this paper does not increase the size of the problem, but is based on the decomposition of the feasible set into subsets, referred to as cells, so that the original problem reduces to a series of linear multiple objective programs over the cells. The approach is an extension of the algorithm proposed by [Nickel, 1995] for multiple-objective planar location problems.

In the next section the MOPLP is formulated and basic concepts are presented. The decomposition of the feasible set is studied in Section 3. The concepts of cell-efficiency and complex-efficiency are introduced in Section 4 and their relationship with efficiency is examined. Section 5 includes a generic algorithm for finding the efficient set of the biobjective piecewise linear program, and an illustrative example is contained in Section 6 . Section 7 concludes the paper.

\section{Definitions and basic concepts}

Consider the following multiple objective piecewise linear program (MOPLP)

$$
\left.\begin{array}{l}
\operatorname{minimize} \\
\text { s.t. } x \in X,
\end{array}\left[f_{1}(x), \ldots, f_{m}(x)\right]\right\} M O P L P
$$

where

$$
\begin{aligned}
f_{i}(x) & =\max _{1 \leq k \leq K_{i}}\left\{f_{i 1}(x), \ldots, f_{i K_{i}}(x)\right\}, i=1, \ldots, m \\
f_{i k}(x) & =\left(c^{i k}\right)^{T} x+d_{i k}, k=1, \ldots, K_{i}
\end{aligned}
$$

$c^{i k}$ is an $n \times 1$ finite vector, $d_{i k}$ is a scalar, $K_{i}$ is the number of affine functions $f_{i k}$ defining the piecewise linear function $f_{i}, X=\left\{x \in \mathbb{R}^{n}: A x=b, x \geq 0\right\}, A$ is an $m \times n$ matrix of full rank, $b$ is an $m \times 1$ vector.

For every $f_{i}, i=1, \ldots, m$, we can derive a subdivision of $X$ by defining cells if

$$
C_{i}^{p_{i}}:= \begin{cases}x \in X: f_{i p_{i}}(x) \geq f_{i k}(x) \text { for } k=1, \ldots, K_{i} & \text { if } \exists x \in X: f_{i p_{i}}(x)>f_{i k}(x) \\ \emptyset \quad \text { otherwise. } & \text { for } k=1, \ldots, K_{i}, k \neq p_{i},\end{cases}
$$

We say that $f_{i p_{i}}$ generates $C_{i}^{p_{i}}$. Then we have

$$
X=\bigcup_{p_{i}=1}^{Q_{i}} C_{i}^{p_{i}}, \quad i=1, \ldots, m
$$

with $\operatorname{dim}\left(C_{i}^{p_{i}}\right)=n$ (because $\operatorname{int}\left(C_{i}^{p_{i}}\right) \neq \emptyset$ ) and $1 \leq Q_{i} \leq k_{i}$. We say that function $f_{i p_{i}}$ is active in a cell $C_{i}^{p_{i}}$ if

$$
f_{i}(x)=f_{i p_{i}}(x) \quad \text { for every } \quad x \in C_{i}^{p_{i}}
$$


For a general introduction to piecewise linear mappings see [Rourke and Sanderson, 1972]. A feasible point $x^{\circ} \in X$ is said to be an efficient solution of the MOPLP if there is no other $x \in X$ such that $f_{i}(x) \leq f_{i}\left(x^{\circ}\right), i=1, \ldots, m$, with at least one strict inequality. Let $X_{E}$ and $X_{P E}$ denote the set of efficient and properly efficient solutions (in the sense of [Geoffrion, 1968]) of MOPLP.

Geometrically related to the concept of efficiency is the concept of level sets and level curves.

Let $y \in \mathbb{R}^{m}$.

$$
\begin{aligned}
& L_{\leq}^{i}\left(y_{i}\right):=\left\{x \in X: f_{i}(x) \leq y_{i}\right\}, i=1, \ldots, m \\
& L_{=}^{i}\left(y_{i}\right):=\left\{x \in X: f_{i}(x)=y_{i}\right\}, i=1, \ldots, m .
\end{aligned}
$$

The following theorem summarizes properties of the sets $X_{E}$ and $X_{P E}$, (see [Geoffrion, 1968], [Gal, 1986], [Hamacher and Nickel, 1993], and [Ehrgott et al., 1996])

Theorem 2.1 1. Let $x^{\circ} \in X$ and $y_{i}^{\circ}=f_{i}\left(x^{\circ}\right), i=1, \ldots, m$. $x^{\circ} \in X_{E}$ iff

$$
\bigcap_{i=1}^{m} L_{\leq}^{i}\left(y_{i}^{\circ}\right)=\bigcap_{i=1}^{m} L_{=}^{i}\left(y_{i}^{\circ}\right) .
$$

2. $X_{E}=X_{P E}$.

3. The set $X_{E}$ is connected.

In the next sections we will use the presented concepts to gain more insight into the structure of the feasible set as well as efficiency with respect to the cells.

\section{Properties of the decomposition}

We start with a property specifiying active functions in the cells.

Theorem 3.1 Let $f(x):=\max \left\{f_{1}(x), \ldots, f_{K}(x)\right\}$ be a piecewise linear (convex) function, where

$$
\begin{aligned}
f_{i}: \mathbb{R}^{n} & \rightarrow \mathbb{R}^{1}, i=1, \ldots, K \\
f_{i}(x) & =\left(c^{i}\right)^{T} x+d_{i},
\end{aligned}
$$

$c^{i}$ is an $n \times 1$ vector and $d_{i}$ is a scalar and $x \in X$, and $X$ is a polyhedral set defined as in Section 2. Let $C^{q}$ be a cell in $X$ so that function $f_{p}, p \in\{1, \ldots, K\}$ is active in it. Then, function $f_{p}$ cannot be active in any other cell in $X$ disjoint with the cell $C^{q}$.

\section{Proof:}

This follows from the fact that the region in which function $f_{1 p}$ is active can be written as $\left\{x \in \mathbb{R}^{n}: f_{1 p}(x) \geq f_{1 i}(x), i=1, \ldots, K\right\}$ which is a polyhedral set. 
Consider now a bi-objective case of the MOPLP:

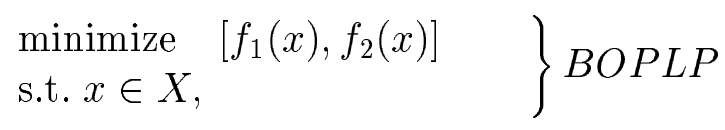

where

$$
\begin{aligned}
f_{1}(x) & :=\max \left\{f_{11}(x), \ldots, f_{1 t}(x), \ldots, f_{1 u}(x), \ldots, f_{1 K}(x)\right\} \\
f_{2}(x) & :=\max \left\{f_{21}(x), \ldots, f_{2 v}(x), \ldots, f_{2 w}(x), \ldots, f_{2 L}(x)\right\}
\end{aligned}
$$

and $X$ is defined as in Section 2. Observe that if $f_{1 t}(x)=f_{2 w}(x)$ for every $x \in X$, then the same function is active in two disjoint cells w.r.t. two (different) criterion indices.

Theorem 3.2 Consider the BOPLP. Let $f_{1 t}(x)=f_{2 w}(x)$ for every $x \in X$. Let $f_{1 t}(x)$ be active in two disjoint cells, say $C^{q}$ and $C^{r}$, w.r.t. the two (different) criterion indices, respectively. Then the other two functions, that are active in $C^{q}$ and $C^{r}$ w.r.t. the other two criterion indices respectively, cannot be the same.

\section{Proof:}

Let $C^{q} \cap C^{r}=\emptyset$. Assume that $f_{1 t}$ and $f_{2 v}$ are active in cell $C^{q}$, and $f_{1 u}$ and $f_{2 w}$ are active in cell $C^{r}$, where

$$
f_{2 w}(x)=f_{1 t}(x) \quad \text { for every } \quad x \in X
$$

Then we have

$$
f_{1 t}(x) \geq f_{1 u}(x) \text { for all } x \in C^{q} \text { and } k=1, \ldots, t, \ldots, u, \ldots, K,
$$

and

$$
f_{1 u}(x) \geq f_{1 k}(x) \text { for all } x \in C^{r} \text { and } k=1, \ldots, t, \ldots, u, \ldots, K \text {. }
$$

Similarly

$$
f_{2 v}(x) \geq f_{2 k}(x) \text { for all } x \in C^{q} \text { and } k=1, \ldots, v, \ldots, w, \ldots, L,
$$

and

$$
f_{1 t}(x) \geq f_{2 k}(x) \text { for all } x \in C^{r} \text { and } k=1, \ldots, v, \ldots, w, \ldots, L \text {. }
$$

Assume now that

$$
f_{2 v}(x)=f_{1 u}(x) \text { for every } \quad x \in X,
$$

and consider points in $C^{q}$ and $C^{r}$. For any $x^{q} \in C^{q}$ and $x^{r} \in C^{r}$ there must be either

$$
f_{1 t}\left(x^{q}\right)>f_{1 t}\left(x^{r}\right)=f_{2 w}\left(x^{r}\right)
$$


or

$$
f_{1 t}\left(x^{q}\right)<f_{1 t}\left(x^{r}\right)=f_{2 w}\left(x^{r}\right) .
$$

Assume the case given by (8).

From (7), (2), (3) and (4) we get

$$
f_{1 t}(x) \geq f_{1 u}(x)=f_{2 v}(x) \geq f_{2 w}(x)=f_{1 t}(x) \quad \text { for any } \quad x \in C^{q} .
$$

Therefore

$$
f_{1 u}(x)=f_{2 w}(x) \text { for any } \quad x \in C^{q} .
$$

From (7), (4) and (6) we get

$$
f_{1 u}(x) \geq f_{1 t}(x) \geq f_{2 v}(x)=f_{1 u}(x) \quad \text { for any } \quad x \in C^{r} .
$$

Therefore

$$
f_{1 t}(x)=f_{2 v}(x) \quad \text { for any } \quad x \in C^{r} \text {. }
$$

(2), (7) and (10) imply

$$
f_{1 t}(x)=f_{1 u}(x)=f_{2 v}(x)=f_{2 w}(x) \text { for any } \quad x \in C^{q},
$$

and (2), (7) and (11) imply

$$
f_{1 t}(x)=f_{1 u}(x)=f_{2 v}(x)=f_{2 w}(x) \text { for any } \quad x \in C^{r} .
$$

Let $h(x)$ be a function defined by (12) and (13). Then $h(x)$ is active in two disjoint cells, $C^{q}$ and $C^{r}$, w.r.t. each criterion index, which by Theorem 3.1 is a contradiction.

In general, the feasible set $X$ can be decomposed into a finite number of cells $C^{q}$ generated by all functions $f_{i}, i=1, \ldots, m$, so that

$$
X=\bigcup_{q=1}^{Q} C^{q},
$$

where $\mathrm{Q}$ is the total number of cells and

$$
f_{i}(x)=f_{i q_{i}}(x) \text { for every } \quad x \in C^{q},
$$

where $q_{i} \in\left\{1, \ldots, K_{i}\right\}, i=1, \ldots, m$.

Let $\mathbf{C}$ denote the set of all cells $C^{q}$ in $X$. In each cell $C^{q} \in C, f(x)$ can be written as a vector $f^{q}$ of $m$ linear functions, $f^{q}=\left(f_{1}^{q}, \ldots, f_{m}^{q}\right)^{T}$. Observe that there are no two distinct cells in $\mathbf{C}$, say $C^{q}$ and $C^{r}$, in which $f^{q}$ and $f^{r}$ are active, respectively, and

$$
f^{q}(x)=f^{r}(x) .
$$


Therefore, the total number $Q$ of cells in $\mathbf{C}$ is

$$
1 \leq Q \leq \prod_{i=1}^{m} K_{i}
$$

Let $\mathcal{F}$ denote a face of a cell. A face $\mathcal{F} \subset C^{q}$ is maximal efficient if there is no other efficient face $\mathcal{F}^{\prime} \subset C^{q}$ so that $\mathcal{F} \subset \mathcal{F}^{\prime}$. The set $C(\mathcal{F}):=\left\{C^{q} \in \mathbf{C}: \mathcal{F} \subset C^{q}\right\}$ is referred to as a cell complex or a complex.

Let $x$ be an extreme point of a cell. Denote $C(x):=\left\{C^{q} \in C: x \in C^{q}\right\}$. Two distinct cells $C^{q}$ and $C^{r}$ are said to be adjacent if there exists a face $\mathcal{F}$ so that $\mathcal{F} \subseteq C^{q} \cap C^{r}$ and $\operatorname{dim}(\mathcal{F}) \geq 1$.

\section{Efficiency with respect to cells}

The set $L_{\leq}^{i}\left(y_{i}\right)$ is a polyhedral set whose defining halfspaces are given by

$$
f_{i k}(x) \leq y_{i}, k=1, \ldots, K_{i}
$$

The set $L_{=}^{i}\left(y_{i}\right)$ represents the set of the hyperplanes associated with the defining halfspaces (16). Let $x^{\circ} \in L_{=}^{i}\left(y_{i}\right)$. There is a finite number of the defining hyperplanes passing through $x^{\circ}$.

Let $K(x)$ denote a cone with origin at point $x$ and $\partial K(x)$ denote its boundary. Define $K^{i}\left(x^{\circ}\right)$ to be the cone generated by the finite number of the defining halfspaces whose associated hyperplanes pass through $x^{\circ}$. Then

$$
L_{\leq}^{i}\left(y^{\circ}\right) \subseteq K^{i}\left(x^{\circ}\right) \text { for every } i=1, \ldots, m
$$

and

$$
\bigcap_{i=1}^{m} L_{\leq}^{i}\left(y^{\circ}\right) \subseteq \bigcap_{i=1}^{m} K^{i}\left(x^{\circ}\right) .
$$

Accordingly

$$
L_{=}^{i}\left(y_{i}^{\circ}\right) \subseteq \partial K^{i}\left(x^{\circ}\right) \text { for every } i=1, \ldots, m
$$

and

$$
\bigcap_{i=1}^{m} L_{\leq}^{i}\left(y^{\circ}\right) \subseteq \bigcap_{i=1}^{m} \partial K^{i}\left(x^{\circ}\right) .
$$

Theorem 4.1 Let $x^{\circ} \in X$ and $y_{i}^{\circ}=f_{i}\left(x^{\circ}\right), i=1, \ldots, m$.

$x^{\circ} \in X_{E}$ iff

$$
\bigcap_{i=1}^{m} K^{i}\left(x^{\circ}\right)=\bigcap_{i=1}^{m} \partial K^{i}\left(x^{\circ}\right) .
$$




\section{Proof:}

$(\Rightarrow)$ If $x^{\circ} \in X_{E}$ than (1) holds. Using (17) and (18) we obtain (19).

$(\Leftarrow)$ Assume that $x^{\circ}$ is not efficient. Then there exists an $x \in X$ such that $f_{i}(x) \leq f_{i}\left(x^{\circ}\right)$ for all $i=1, \ldots, m$, and for at least one index $k, k \in\{1, \ldots, m\}, f_{k}(x)<f_{k}\left(x^{\circ}\right)$. Therefore $x \in L_{\leq}^{i}\left(y_{i}^{\circ}\right)$ for all $i=1, \ldots, m$, where $y_{i}^{\circ}=f_{i}\left(x^{\circ}\right)$ and $x \notin L_{=}^{k}\left(y_{i}^{\circ}\right)$ for at least one index $k$. Consequently $x \in \bigcap_{i=1}^{m} L_{\leq}^{i}\left(y_{i}^{\circ}\right)$ and, using (17) we get

$$
x \in \bigcap_{i=1}^{m} K^{i}\left(x^{\circ}\right) .
$$

Since $x \notin L_{=}^{k}\left(y_{i}^{\circ}\right)$ then

$$
x \notin \partial K^{k}\left(x^{\circ}\right)
$$

From (20) and (21) we conclude a contradiction.

Let $x^{\circ} \in C^{q}$. Since $f_{i}(x)=f_{i p_{i}}(x), p_{i} \in\left\{1, \ldots, K_{i}\right\}$, for every $x \in C^{q}$, the set $L_{\leq}^{i}\left(y_{i}^{\circ}\right)$ includes exactly one defining halfspace

$$
H_{\leq}^{i, q}\left(x^{\circ}\right)=\left\{x \in X: f_{i p_{i}}(x) \leq y_{i}^{\circ}\right\}
$$

whose associated hyperplane $H_{\stackrel{i, q}{=}}\left(x^{\circ}\right)$ passes through $x^{\circ}$ and the set $C^{q} \cap L_{=}^{i}\left(y_{i}^{\circ}\right)$.

Definition $4.1 x^{\circ} \in C^{q}$ is said to be cell-efficient if

$$
\bigcap_{i=1}^{m} H_{\leq}^{i, q}\left(x^{\circ}\right)=\bigcap_{i=1}^{m} H_{=}^{i, q}\left(x^{\circ}\right) .
$$

Theorem 4.2 Let $x^{\circ} \in \operatorname{int}\left(C^{q}\right)$. A point $x^{\circ} \in X_{E}$ iff $x^{\circ}$ is cell-efficient.

\section{Proof:}

$(\Rightarrow)$ the proof is obvious

$(\Leftarrow)$ If $x^{\circ}$ is cell-efficient then $(23)$ holds. Also $K^{i}\left(x^{\circ}\right)=H_{\leq}^{i, q}\left(x^{\circ}\right)$ and $\partial K^{i}\left(x^{\circ}\right)=H_{\stackrel{i, q}{=}}\left(x^{\circ}\right)$. Therefore, using (1), $x^{\circ}$ is efficient.

Corollary 4.1 Let $x^{\circ} \in \operatorname{int}\left(C^{q}\right)$. If $x^{\circ}$ is cell-efficient, then $C^{q} \in X_{E}$.

Let $C(\mathcal{F})=\left\{C^{q}, q=1, \ldots, r\right\}$.

Definition 4.2 Let $x^{\circ} \in \operatorname{rel} \operatorname{int}(\mathcal{F})$. A point $x^{\circ}$ is said to be complex-efficient if it is cell-efficient for every cell $C^{q} \in C(\mathcal{F})$. 
Theorem 4.3 Let $x^{\circ} \in \operatorname{rel} \operatorname{int}(\mathcal{F})$ and $C(\mathcal{F})$ be a cell complex containing the face $\mathcal{F}$. $A$ point $x^{\circ} \in X_{E}$ iff $x^{\circ}$ is complex-efficient.

\section{Proof:}

$(\Rightarrow)$ the proof is obvious

$(\Leftarrow)$ If $x^{\circ}$ is complex-efficient, then it is cell-efficient for every cell $C^{q} \in C(\mathcal{F})$. That is

$$
\bigcap_{i=1}^{m} H_{\leq}^{i, q}\left(x^{\circ}\right)=\bigcap_{i=1}^{m} H_{=}^{i, q}\left(x^{\circ}\right) \quad \text { for all } \quad q=1, \ldots, r .
$$

Therefore

$$
\bigcap_{q=1}^{r}\left(\bigcap_{i=1}^{m} H_{\leq}^{i, q}\left(x^{\circ}\right)\right)=\bigcap_{q=1}^{r}\left(\bigcap_{i=1}^{m} H_{=}^{i, q}\left(x^{\circ}\right)\right),
$$

which implies

$$
\bigcap_{i=1}^{m}\left(\bigcap_{q=1}^{r} H_{\leq}^{i, q}\left(x^{\circ}\right)\right)=\bigcap_{i=1}^{m}\left(\bigcap_{q=1}^{r} H_{=}^{i, q}\left(x^{\circ}\right)\right) .
$$

Now observe that since $x \in C^{q}$ for all $q=1, \ldots, r$, the cone $K_{i}\left(x^{\circ}\right)$ is defined by exactly $r$ halfspaces $H_{\leq}^{i, q}\left(x^{\circ}\right)$. We get

$$
K_{i}\left(x^{\circ}\right)=\bigcap_{q=1}^{r} H_{\leq}^{i, q}\left(x^{\circ}\right)
$$

and

$$
\partial K_{i}\left(x^{\circ}\right)=\bigcap_{q=1}^{r} H_{=}^{i, q}\left(x^{\circ}\right) .
$$

From (24), (25), (26) and (1) we conclude that $x^{\circ} \in X_{E}$.

Theorem 4.4 Let $x^{\circ} \in \operatorname{rel} \operatorname{int}(\mathcal{F})$. If $x^{\circ}$ is complex-efficient then the face $\mathcal{F} \in X_{E}$.

\section{Proof:}

Let $\hat{x} \in \mathcal{F}$ and $\hat{x} \neq x^{\circ}$. Assume that $\hat{x}$ is not efficient, so it is not complex-efficient either. There exists at least one cell $C^{t} \in C(\mathcal{F}), t \in\{1, \ldots, r\}$, such that $\hat{x}$ is not cell-efficient. By Definition 4.1.

$$
\bigcap_{i=1}^{m} H_{\leq}^{i, t}(\hat{x}) \neq \bigcap_{i=1}^{m} H^{i, t}(\hat{x}) .
$$

Since $x^{\circ} \in \mathcal{F}$, then $x^{\circ} \in C^{t}$ and since $x^{\circ}$ is efficient

$$
\bigcap_{i=1}^{m} H_{\leq}^{i, t}\left(x^{\circ}\right)=\bigcap_{i=1}^{m} H^{i, t}\left(x^{\circ}\right)
$$

Observe that $\bigcap_{i=1}^{m} H_{\leq}^{i, t}(\hat{x})$ and $\bigcap_{i=1}^{m} H_{\leq}^{i, t}\left(x^{\circ}\right)$ determine the same cones with distinct origins, and co-existence of (27) and (28) is a contradiction. 


\section{Algorithm}

The results of the previous sections lead to the development of an approach to finding the efficient set $X_{E}$ of the BOPLP. We now present this approach.

First observe that the lexicographic optimal solutions $X_{i}^{l e x}, i=1,2$, of the BOPLP are in $X_{E}$, and since $X_{E}$ is connected, it must consist of a chain of efficient cells and/or faces of cells "spanned" in the feasible set between the sets of lexicographic solutions. $X_{i}^{l e x}, i=1,2$, can be found solving the following single objective problems

$$
\begin{aligned}
\operatorname{minimize} & f_{j}(x) \\
\text { s.t. } x \in X_{i}^{*}, & i \neq j,
\end{aligned}
$$

where $X_{i}^{*}$ is the optimal solution set of the problem

$$
\begin{aligned}
& \operatorname{minimize} \\
& \text { s.t. } x \in X .
\end{aligned}
$$

Given an extreme point in $X_{i}^{l e x}$, an efficient cell (or face) adjacent to that point is identified. Then a new extreme point, which belongs to that efficient cell (or face) and at which the objective function $f_{j}, j \neq i$, assumes the smallest value over the whole cell (or face), is found. At the extreme points subsequently found, the efficiency test for a new cell (or face) is performed until a newly found extreme point is in $X_{j}^{l e x}, j \neq i$. Then the complete efficient set is generated.

Below we present this approach in generic form.

\section{Initialization:}

1. For $i=1,2$ solve:

$$
\begin{aligned}
& \operatorname{minimize} \\
& \text { s.t. } x \in X .
\end{aligned}
$$

Let $X_{i}^{*}$ be the set of optimal solutions.

2. For $j=1,2$ solve

$$
\begin{aligned}
\operatorname{minimize} & f_{j}(x) \\
\text { s.t. } x \in X_{i}^{*}, & i \neq j .
\end{aligned}
$$

Let $X_{i}^{\text {lex }}$ be the set of optimal solutions. 
3. Set $X_{E}:=\bigcup_{i=1}^{2} X_{i}^{l e x}$.

4. Let $x \in X_{1}^{\text {lex }}$. If $x$ is an extreme point of a cell, go to the main step. Otherwise $x \in \operatorname{rel} \operatorname{int}(\mathcal{F})$ and use a procedure for finding an extreme point of $X_{1}^{\text {lex }}$. Then go to the main step.

\section{Main Step:}

Let $y \in X_{1}^{\text {lex }}$ be an efficient extreme point of a cell $C^{q}$. while $y \notin X_{2}^{\text {lex }}$ do

- Find $C(y)$.

- If there is an efficient cell $C^{r} \subset C(y)$, set $X_{E}:=X_{E} \cup C^{r}$, find $z=\arg \underset{x \in C^{r}}{\min } f_{2}(x)$, and set $y:=z$.

- Otherwise find a maximal efficient face $\mathcal{F}$ such that $y \in \mathcal{F} \subset C(y)$, set $X_{E}:=X_{E} \cup \mathcal{F}$, find $z=\arg \min _{x \in \mathcal{F}} f_{2}(x)$ and set $y:=z$.

end

Output: $X_{E}$

The approach includes four major tasks, namely

i) find an extreme point in $X_{i}^{l e x}$,

ii) find a cell complex $C(y)$,

iii) perform efficiency test for a cell,

iv) perform efficiency test for a face,

which we will now discuss in more detail.

\section{Procedures for finding an extreme point in $X_{i}^{l e x}$}

\section{Procedure A:}

1. After performing step 1 . of the initialization, enumerate all extreme points in $X_{i}^{*}$. Let $\operatorname{EP}\left(X_{i}^{*}\right)$ be the set of all extreme points in $X_{i}^{*}$. 
2. Perform step 2. of the initialization as follows:

$$
\begin{aligned}
\operatorname{minimize} & f_{j}(x) \\
\text { s.t. } x \in \operatorname{EP}\left(X_{i}^{*}\right), & i \neq j .
\end{aligned}
$$

Output: an extreme point in $X_{i}^{l e x}$.

Procedure B: (see [Bazaraa et al., 1990])

Let $\mathcal{F}$ be a face of a cell $C^{q}$ and $x \in \operatorname{rel} \operatorname{int}(\mathcal{F})$. Let the system $H x=h$ represent the hyperplanes binding at $x$. Note that $\operatorname{rank}(H) \leq n-1$. Find a solution $d \neq 0$ to the system $H d=0$ and compute $\gamma_{1}=\max \{\gamma: x+\gamma d \in \mathcal{F}\}<\infty$. Let $y^{1}=y+\gamma_{1} d$. Hence at $y^{1}$ there is at least one additional linearly independent hyperplane binding. If the new binding hyperplane(s) along with $H x=h$ produce a system of rank $n$, then $y^{1}$ is an extreme point of the cell $C^{q}$. Otherwise, repeat this step at $y^{1}$ until after at most $n$-rank $(H)$ such steps, an extreme point of $C^{q}$ satisfying $H y^{1}=g$ is obtained.

Output: an extreme point in $X_{i}^{l e x}$.

\section{Cell complex $C(y)$}

Finding $C(y)$ just involves identifying the indices of the functions active in all the cells adjacent to the extreme point $y$.

Efficiency test for a cell $C^{q}$ (based on Theorem 4.2 and Corollary 4.1)

Let a cell $C^{q}$ be given and let the functions $f_{1 t}$ and $f_{2 v}$ be active in $C^{q}$, where

$$
\begin{aligned}
& f_{1 t}(x)=\left(c^{1 t}\right)^{T} x+d_{1 t} \\
& f_{2 v}(x)=\left(c^{2 v}\right)^{T} x+d_{2 v} .
\end{aligned}
$$

If the vectors $c^{1 t}$ and $c^{2 v}$ are a negative linear combination of each other then the whole cell $C^{q}$ is efficient. Note that $c^{1 t}=0$ or $c^{2 v}=0$ means that we are in the set of optimal solutions for $f_{1}$ or $f_{2}$, respectively.

Efficiency test for a face $\mathcal{F}$ (based on Theorem 4.4)

This test is performed if there is no efficient cell in $C(y)$.

Let $G^{q}$ denote the $2 \times n$ matrix whose rows are composed of the gradients of the objective functions active in the cell $C^{q}$. The matrix $G^{q}$ will be referred to as the gradient matrix of the cell $C^{q}$. 
In order to check the efficiency of an $n-1$ dimensional face in $C(y)$, construct the linear system:

$$
\left\{\begin{array}{l}
G^{q} d \leq 0 \\
G^{r} d \leq 0
\end{array}\right.
$$

where $G^{q}$ and $G^{r}$ are the gradient matrices of two adjacent cells $C^{q}$ and $C^{r}$ in $C(y)$, respectively. Solve system $(29)$ for $d \neq 0$. If system (29) is infeasible, then the face being the intersection of $C^{q}$ and $C^{r}$ is efficient. In fact, it is a maximal efficient face adjacent to $y$. Otherwise, that face is not efficient and one should search for $n-2$ dimensional efficient faces in $C(y)$. In general, in order to perform the efficiency test for an $n-k$ dimensional face in $C(y)$ construct the linear system:

$$
\left\{\begin{aligned}
G^{1} d & \leq 0 \\
\vdots & \\
G^{k+1} d & \leq 0
\end{aligned}\right.
$$

where $G^{j}, j=1, \cdots, k+1$, are the gradient matrices of the adjacent cells $C^{j}$ in $C(y)$, respectively.

If system (30) is infeasible, then the face being the intersection of $C^{j}, j=1, \cdots, k+1$ is efficient. Otherwise proceed to examining faces of a lower dimension.

Observe, that for some $k, k=1, \cdots, n-1$, system (30) has to be infeasible implying that the corresponding $n-k$ dimensional face is efficient.

\section{Example}

Consider the following BOPLP:

$$
\begin{aligned}
& \operatorname{minimize}\left[\begin{array}{c}
\max _{i=1, \cdots, 5}\left\{f_{1 i}(x)\right\} \\
\max _{i=1, \cdots, 5}\left\{f_{2 i}(x)\right\}
\end{array}\right] \\
& \text { s.t. } x \in X=\left\{x \in \mathbb{R}^{2}: x \geq 0\right\},
\end{aligned}
$$

where

$$
\begin{array}{ll}
f_{11}=x_{1}+x_{2}-20 & f_{21}=x_{1}+x_{2}-60 \\
f_{12}=x_{1}-x_{2} & f_{22}=x_{1}-x_{2}-20 \\
f_{13}=-x_{1}+x_{2} & f_{23}=-x_{1}+x_{2}+20 \\
f_{14}=-x_{1}-x_{2}+20 & f_{24}=-x_{1}-x_{2}+60 \\
f_{15}=\frac{1}{2} x_{1}+10 & f_{25}=-\frac{1}{2} x_{1}+35 .
\end{array}
$$

Observe that

$$
X_{1}^{*}=X_{1}^{l e x}=\left\{\left(\begin{array}{c}
0 \\
10
\end{array}\right)\right\}
$$




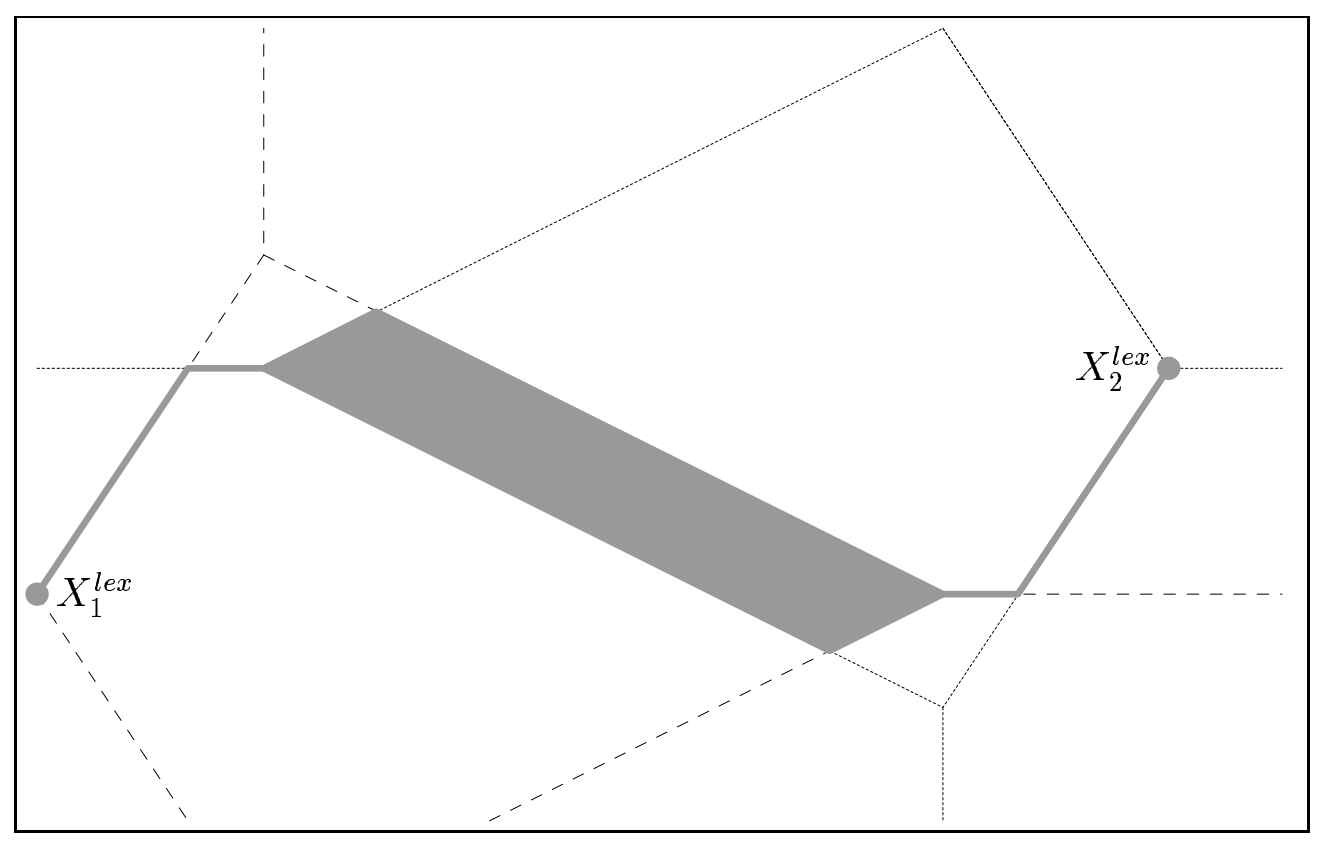

Figure 1: Illustration for the example. The gray part is the efficient set. The dashed and dotted lines represent the cell subdivision given by $f_{1}$ and $f_{2}$, respectively.

and

$$
X_{2}^{*}=X_{2}^{\text {lex }}=\left\{\left(\begin{array}{l}
50 \\
20
\end{array}\right)\right\} .
$$

The efficient set is the union of 4 maximal efficient faces and one efficient cell.

$$
X_{E}=\mathcal{F}_{1} \cup \mathcal{F}_{2} \cup C^{1} \cup \mathcal{F}_{3} \cup \mathcal{F}_{4},
$$

where

$$
\begin{aligned}
& \mathcal{F}_{1}=\operatorname{conv}\left(\left(\begin{array}{c}
0 \\
10
\end{array}\right),\left(\begin{array}{c}
6.6 \\
20
\end{array}\right)\right) \\
& \mathcal{F}_{2}=\operatorname{conv}\left(\left(\begin{array}{c}
6.6 \\
20
\end{array}\right),\left(\begin{array}{c}
10 \\
20
\end{array}\right)\right), \\
& C^{1}=\operatorname{conv}\left(\left(\begin{array}{c}
10 \\
20
\end{array}\right),\left(\begin{array}{c}
15 \\
22.5
\end{array}\right),\left(\begin{array}{c}
35 \\
7.5
\end{array}\right),\left(\begin{array}{c}
40 \\
10
\end{array}\right)\right), \\
& \mathcal{F}_{3}=\operatorname{conv}\left(\left(\begin{array}{c}
40 \\
10
\end{array}\right),\left(\begin{array}{c}
43.3 \\
10
\end{array}\right)\right) \\
& \mathcal{F}_{4}=\operatorname{conv}\left(\left(\begin{array}{c}
43.3 \\
10
\end{array}\right),\left(\begin{array}{c}
50 \\
20
\end{array}\right)\right),
\end{aligned}
$$

and $\operatorname{conv}\left(x^{1}, \ldots, x^{n}\right)$ denotes the convex hull of $\left\{x^{1}, \ldots, x^{n}\right\}$.

The feasible set, cells, and efficient set of this problem are depicted in Figure 1. 


\section{Conclusions}

In this paper multiple objective piecewise linear programs (MOPLPs) are studied and an approach to generating their efficient set is proposed. The new concepts of cell-efficiency and complex-efficiency help examine the efficiency of solutions. The approach is based on the decomposition of the feasible set into cells so that the efficient set is available as the union of efficient cells and/or maximal efficient faces.

A generic method for finding the efficient set of bi-objective piecewise linear programs is presented. We emphasize that while other methods of generating the efficient set of multiple objective linear programs usually start with identifying efficient extreme points and then use this information to identify maximal efficient faces, the proposed method directly finds efficient cells and/or maximal efficient faces. Due to this fact, we expect that this approach is computationally more attractive, however implementation studies are needed to verify this hypothesis. When extended to problems with multiple objective functions, the approach will have the same structure although some modifications will be needed due to a bigger number of the objective functions.

\section{Acknowledgements}

The authors would like to express their acknowledgements to Ansgar Weißler for preparing the numerical example.

\section{References}

[Achary, 1989] Achary, K. K. (1989). A direct simplex method for determining all efficient solution pairs of time-cost transportation problem. Economic Computation and Economic Cybernetics Studies and Research, 2(2/3):139-150.

[Armand, 1993] Armand, P. (1993). Finding all maximal efficient faces in multiobjective linear programming. Mathematical Programming, 61:357-375.

[Bazaraa et al., 1990] Bazaraa, M., Jarvis, J., and Sherali, H. (1990). Linear Programming and Network Flows. John Wiley, New York.

[Burkard et al., 1991] Burkard, R. E., Hamacher, H. W., and Rote, G. (1991). Sandwich approximation of univariate convex functions with an application to separable convex programming. Naval Research Logistics Quarterly, 38:911-924.

[Durier and Michelot, 1986] Durier, R. and Michelot, C. (1986). Set of efficient points in a normed space. Journal of Mathematical Analysis and Applications, 117:506-528.

[Ecker et al., 1980] Ecker, J., Hegner, N., and Kouada, I. (1980). Generating all maximal efficient faces for multiple objective linear programs. Journal of Optimization Theory and Applications, 30:353-381. 
[Ehrgott et al., 1996] Ehrgott, M., Hamacher, H., Klamroth, K., Nickel, S., Schoebel, A., and Wiecek, M. (1996). A note on the equivalence of balance points and pareto solutions in multiple objective programming. Journal of Optimization Theory and Applications. To appear.

[Fourer, 1985] Fourer, R. (1985). A simplex algorithm for piecewise-linear programming I: derivation and proof. Mathematical Programming, 33:204-233.

[Fourer, 1988] Fourer, R. (1988). A simplex algorithm for piecewise-linear programming II: finiteness, feasibility and degeneracy. Mathematical Programming, 41:281-315.

[Fourer, 1992] Fourer, R. (1992). A simplex algorithm for piecewise-linear programming III: computational analysis and applications. Mathematical Programming, 53:213-235.

[Gal, 1977] Gal, T. (1977). A general method for determining the set of all efficient solutions to a linear vector maximum problem. European Journal of Operational Research, $1: 307-322$.

[Gal, 1986] Gal, T. (1986). On efficient sets in vector maximum problems - a brief survey. European Journal of Operational Research, 24:253-264.

[Geoffrion, 1968] Geoffrion, A. M. (1968). Proper efficiency and the theory of vector maximization. Journal of Mathematical Analysis and Applications, 22:618-630.

[Haimes and Chankong, 1983] Haimes, Y. and Chankong, V. (1983). Multiobjective Decision Making - Theory and Methodology. North-Holland, New York.

[Hamacher and Nickel, 1993] Hamacher, H. W. and Nickel, S. (1993). Multicriterial planar location problems. Technical Report 243, Department of Mathematics, University of Kaiserslautern. Accepted by European Journal of Operational Research.

[Isermann, 1977] Isermann, H. (1977). The enumeration of the set of all efficient solutions for a linear multiple objective program. Operational Research Quarterly, 28:711-725.

[Murty, 1983] Murty, K. (1983). Linear Programming. John Wiley, New York.

[Nickel, 1995] Nickel, S. (1995). Discretization of Planar Location Problems. PhD thesis, Fachbereich Mathematik, Universitaet Kaiserslautern, Germany.

[Pelegrin and Fernandez, 1988] Pelegrin, B. and Fernandez, F. (1988). Determination of efficient solutions for point objective locational decision problems. Naval Research Logistics, 35:697-705.

[Puerto and Fernandez, 1994] Puerto, J. and Fernandez, F. (1994). Multicriteria decisions in location. Studies in Locational Analysis, 7:185-199.

[Rourke and Sanderson, 1972] Rourke, C. and Sanderson, B. (1972). Introduction to piecewise-linear topology. Springer-Verlag. 
[Steuer, 1986] Steuer, R. (1986). Multiple Criteria Optimization: Theory, Computation and Application. John Wiley, New York.

[Wiecek and Zhang, 1996] Wiecek, M. and Zhang, H. (1996). A parallel algorithm for multiple objective linear programs. Computational Optimization and Applications. To appear.

[Yu and Zeleny, 1975] Yu, P. and Zeleny, M. (1975). The set of non-dominated solutions in linear cases and a multicriteria simplex method. Journal of Mathematical Analysis and Applications, 49:430-468. 